\title{
Consenso sobre terminología y conceptos de la función del tracto urinario inferior
}

\author{
Grupo Español de Urodinámica y de SINUG
}

Actas Urol Esp 2005; 29 (1): 16-30

\section{INTRODUCCIÓN}

Este informe de estandarización está pensado para todo tipo de pacientes (incluyendo todos los grupos de edad) y presenta las definiciones de los sintomas, signos, hallazgos urodinámicos y sus condiciones asociadas a la disfunción del tracto urinario inferior (DTUI) y los estudios urodinámicos.

Estas definiciones presentadas por la Continence Society Standarization of Terminology repiten o actualizan los trabajos previos:

- Terminología del 1 al 7 [Abrams et al. 1988; Abrams et al. 1986; Andersen et al. 1992; Bump et al. 1996; Griffiths et al. 1997; Stohrer et al. 1999] [van Waalwijk et al. 2000]

- 8 función uretral [Lose et al. 2002]

- 9 nocturia [van Kerrebroeck et al. 2002]

- 10 aspectos técnicos del equipo de urodinámica [Rowan et al. 1987]

- 11 informe sobre práctica urodinámica [Schafer et al. 2002]

- 12 al 15 informes de resultados finales [Mattiasson et al. 1998; Lose et al. 1998; Nordling et al. 1998; Fonda et al. 1998]

Se indican las definiciones nuevas y las que han cambiado. No se incluye las recomendaciones referentes a las técnicas.

Las definiciones son compatibles con las de la OMS (International Classification of functioning disability and Health[OMS 2001]) y con las de la International Classification of Diseases (ICD10).

Se pretende, en lo posible, que las definiciones describan lo observado, sin asumir presunciones que podrían ser incorrectas o incompletas.

Con estos principios la ICS trata de hacer posible la comparación de resultados consiguiendo una comunicación.

En este informe se incluyen las siguientes categorías: signos, síntomas y condiciones; se añade la categoría de hallazgos urodinámicos y la terminología propia de los tratamientos [Andersen, Blaivas, Cardozo, and Thüroff 1992].

Cuando se hace referencia a la vejiga urinaria como órgano completo, el término correcto es vejiga. Cuando se hace referencia al músculo liso de la vejiga específicamente, el término correcto es detrusor).

Se aconseja que cuando se realice un trabajo con esta estandarización, se indique en Material y Métodos: "Métodos, definiciones y unidades de acuerdo con la terminología y conceptos recomendados por la ICS, excepto cuando se especifique".

AREAS GUE SE DESARROLLAN EN ESTE TRABAJO

- Síntomas

- Signos

- Hallazgos urodinámicos

- Condiciones

- Tratamiento

Síntomas del Tracto Urinario Inferior (STUI) [Lower Urinary Tract Symptoms (LUTS)]

Los sintomas son el indicador subjetivo que aqueja el enfermo de cambio y/o alteración tal y como lo percibe el paciente, cuidador o pareja y que pueden inducirle a buscar ayuda en los profesionales de la salud (NUEVA).

Los síntomas son el indicador subjetivo de la enfermedad, de los cambios percibidos por el paciente, facilitando al cuidador o al compañero dar los cuidados profesionales correctos.

Los sintomas pueden ser el motivo especifico de consulta o aparecer durante la anamnesis. Suelen ser cualitativos. Generalmente no nos son útiles para realizar un diagnóstico definitivo. También pueden sugerirnos otras patologías distintas de la disfunción del tracto urinario inferior, tales como la infección. 
Signos sugestivos de Disfunción del Tracto Urinario Inferior (DTUI) [Lower Urinary Tract Dysfunction (LUTD)]

Los signos son los indicadores objetivos de enfermedad observados por el médico, incluyendo recursos simples diseñados para verificar y cuantificar los síntomas,_(Nuevo)

Un ejemplo seria la objetivación de escape con la tos. Las hojas de frecuencia/volumen, la prueba del absorbente y cuestionarios validados de síntomas y calidad de vida, son ejemplos de otros instrumentos que pueden ser utilizados para verificar y cuantificar los sintomas.

\section{Hallazgos urodinámicos}

Son las observaciones realizadas durante los estudios urodinámicos (NUEVA).

Un ejemplo sería una contracción involuntaria del detrusor. Generalmente, las observaciones urodinámicas pueden tener diferentes causas, $\mathrm{y}$ no constituyen ellas mismas un diagnóstico de enfermedad o alteración. Pueden ocurrir acompañadas o no de signos y/o síntomas.

\section{Condición}

Se define como la asociación entre hallazgos urodinámicos y síntomas o signos característicos. También se considera condición la evidencia no urodinámica de procesos patológicos relevantes. (NUEVA).

\section{Tratamiento}

El tratamiento de la disfunción del TUI que se asume como válida, está definido en el $7^{\circ}$ trabajo de la ICS sobre técnicas de rehabilitación del tacto urinario inferior [Andersen, Blaivas, Cardozo, and Thüroff1992].

\section{SÍNTOMAS DEL TRACTO URINARIO INFERIOR (STUI)}

Los síntomas del TUI se dividen en tres grupos:

- síntomas de llenado,

- miccionales o de vaciado y

- postmiccionales.

\section{SÍNTOMAS DE LLENADO}

Son los que se perciben durante la fase de llenado vesical e incluyen frecuencia diurna y nocturia (NUEVA)

Frecuencia diurna aumentada cuando el paciente manifiesta que sus micciones son muy frecuentes durante el día (NUEVA). Este término es equivalente a polaquiuria en muchos países.

Nocturia cuando el individuo se queja de que precisa despertarse durante la noche una o más veces para orinar (NUEVA) ${ }^{1}$.

Urgencia: cuando el paciente se queja de la aparición súbita de un deseo miccional claro e intenso difícil de demorar.

Incontinencia urinaria es la manifestación por parte del paciente de escape de orina (NUEVA) ${ }^{2}$.

En cada circunstancia concreta, la incontinencia urinaria debería ser descrita además especificando los factores relevantes como el tipo, frecuencia, severidad, factores desencadenantes, impacto social, efecto sobre la higiene y calidad de vida, las medidas utilizadas para las pérdidas, y si el individuo desea o no ayuda para su incontinencia urinaria ${ }^{3}$.

El escape urinario tiene que distinguirse del sudor o el flujo vaginal.

\footnotetext{
${ }^{1}$ Frecuencia nocturna es la necesidad de orinar una o más veces durante la noche en el tiempo comprendido desde que el individuo se acuesta con la intención de dormir y el momento del despertar con la intención de levantarse. La frecuencia nocturna se diferencia de la nocturia en que aquella incluye las micciones que se realizan después de que el individuo se haya acostado pero antes de haberse quedado dormido; y las micciones de la madrugada si se despierta y no puede volver a dormirse como desearía. Estas micciones antes y después del sueño han de ser también valoradas en el diagnóstico diferencial, por ejemplo, en la poliuria nocturna. Si se usa esta definición sería necesario en consecuencia adaptar la definición de frecuencia diurna.

2 En los escolares y niños pequeños la definición de Incontinencia Urinaria no es aplicable. En las comunicaciones científicas la definición de incontinencia en niños necesita de una explicación añadida.

${ }^{3}$ La definición de incontinencia de la ICS "Incontinencia urinaria es la pérdida involuntaria de orina que genera un problema higiénico o social” la relaciona con la calidad de vida (CdV). Algunos instrumentos de CdV han sido y se están desarrollando para valorar el impacto sobre la calidad de vida tanto de la incontinencia como de otros síntomas del TUS.
} 
- Incontinencia urinaria de esfuerzo es la percepción del escape de orina con el esfuerzo (como por ejemplo tos, estornudo, etc) ${ }^{4}$. (CAMBIADA)

- Incontinencia urinaria de urgencia es la pérdida involuntaria de orina acompañada o inmediatamente precedida de urgencia $^{5}$. (CAMBIADA)

- Incontinencia urinaria mixta es la percepción de pérdida involuntaria de orina asociada tanto a la urgencia como al esfuerzo. (NUEVA)

- Enuresis significa cualquier pérdida de orina (ORIGINAL). Si se usa para denotar incontinencia durante el sueño, debería calificarse siempre con el adjetivo "nocturna"

- Enuresis nocturna es la pérdida de orina que ocurre durante el sueño. (NUEVA)

- Incontinencia urinaria continua es la permanente pérdida de orina. (NUEVA)

- Otros tipos de incontinencia urinaria pueden ocurrir en determinadas situaciones, por ejemplo la incontinencia durante la relación sexual, o la incontinencia con la risa

- La sensación vesical puede ser definida, durante la historia clínica, encuadrándola en cinco categorías:

Normal: Se es consciente del llenado vesical, aumentando esta sensación de forma progresiva, hasta un fuerte deseo miccional. (NUEVA)

Aumentada: se percibe un deseo miccional temprano y persistente. (NUEVA)

Reducida: se es consciente del llenado vesical pero no se percibe un claro deseo de orinar. (NUEVA)

Ausente: no se tiene la sensación del llenado vesical ni deseo miccional. (NUEVA)

Inespecífica: no se tienen las sensaciones normales pero se puede percibir el llenado vesical como una plenitud abdominal, síntomas vegetativos o espasticidad ${ }^{6}$. (NUEVA)

\section{SÍNTOMAS DE VACIADO}

Son los que se perciben durante esta fase. (NUEVA)

- El chorro débil/lento cuando se percibe una reducción del flujo miccional, normalmente referido a situaciones previas o en comparación con otros. (NUEVA)

- Micción en regadera, dispersa o en sifón (NUEVA)

- Chorro intermitente es el término usado cuando el individuo describe un flujo urinario con interrupciones. (NUEVA)

- Retardo dificultad en el inicio de la micción, resultando un retraso en la aparición del chorro miccional desde el momento en que ya se ha decidido orinar. (NUEVA)

- Esfuerzo miccional describe la contracción muscular que se realiza para iniciar, mantener o mejorar el flujo miccional ${ }^{7}$. (NUEVA)

- Goteo terminal describe la prolongación del final de la micción, cuando el débil flujo se transforma en goteo. (NUEVA)

\section{SÍNTOMAS POSTMICCIONALES}

Son los percibidos inmediatamente tras la micción. (NUEVA)

- Sensación de vaciado incompleto es un término autoexplicativo para una sensación percibida por el individuo tras orinar. (NUEVA)

- Goteo postmiccional este término define la perdida involuntaria de orina en forma de goteo, inmediatamente tras la finalización del chorro miccional y usualmente tras haber dejado el W.C. o a la salida. (NUEVA)

\section{SÍNTOMAS ASOCIADOS A LAS RELACIONES SEXUALES}

Las mujeres describen con frecuencia dispareunia, sequedad vaginal e incontinencia durante

\footnotetext{
${ }^{4}$ El término de "incontinencia de estrés" no es satisfactorio debido a sus connotaciones mentales. En Español, Sueco, Francés e Italiano es preferible la expresión "incontinencia de esfuerzo". Sin embargo, en inglés la palabra "effort" no engloba algunos de los factores desencadenantes de incontinencia urinaria como el esfuerzo abdominal, la tos o el estornudo. Por esta razón el término "stress" se deja sin cambios en inglés.

${ }^{5}$ La incontinencia de urgencia presenta diferentes formas sintomáticas, por ejemplo, pérdidas más o menos frecuentes entre las micciones, o como un episodio dramático de micción involuntaria con vaciado del contenido completo de la vejiga.

${ }^{6}$ Los sintomas inespecíficos se ven más frecuentemente en pacientes neurológicos, particularmente en los lesionados medulares y en niños y adultos con malformaciones de la médula espinal.

${ }^{7}$ La presión suprapúbica puede usarse para iniciar o mantener la micción. La maniobra de Credé (presión sobre la zona suprapúbica) es utilizada por pacientes con lesión medular y mujeres con detrusor hipoactivo para facilitar el vaciado.
} 
la relación sexual o tras el coito. Estos síntomas deben ser descritos minuciosamente. Es importante especificar si el escape se produce durante la penetración, el coito, el orgasmo, o tras él.

\section{SÍNTOMAS ASOCIADOS CON EL PROLAPSO PELVIANO}

Las mujeres con prolapso pueden referir la sensación de bulto ("algo que se sale"), sacralgia, pesadez, sensación de llevar algo colgando y/o la necesidad de reponer el prolapso digitalmente para defecar $\mathrm{u}$ orinar.

\section{DOLOR GENITAL O/Y DEL TRACTO URINARIO INFERIOR $^{8}$}

Dolor, molestias y presión son parte de un espectro de sensaciones anormales percibidas por el individuo. El dolor produce el mayor impacto sobre el paciente y puede relacionarse con el llenado o vaciado vesical, puede notarse tras la micción o ser continuo. Debería ser caracterizado por tipo, frecuencia, duración, factores que lo desencadenan o lo atenúan y localización, como se define a continuación:

- Dolor vesical supra o retropúbico, se suele incrementar con el llenado y puede persistir tras la micción. (NUEVA)

- Dolor uretral el individuo indica el trayecto uretral como el lugar doloroso. (NUEVA)

- Dolor vulvar percibido dentro y alrededor de los genitales externos. (NUEVA)

- Dolor vaginal percibido internamente, por dentro del introito. (NUEVA)

- Dolor escrotal puede estar o no localizado, al referirse tanto hacia el testículo como al epidídimo, estructuras del cordón o piel escrotal. (NUEVA)

- Dolor perineal en la mujer, entre la horquilla vulvar posterior (labio posterior del introito) y el ano, y en el varón, entre el escroto y el ano. (NUEVA)

- Dolor pelviano está menos definido que, por ejemplo, el vesical, uretral o perineal y está menos clara la relación con el ciclo miccional o la función intestinal. No está localizado como referido a ningún órgano único pelviano. (NUEVA)

SÍNDROMES DE SÍNTOMAS Y DOLORES GENITO-URINARIOS SUGESTIVOS DE DISFUNCIÓN DEL TRACTO URINARIO INFERIOR (DTUI)

En las comunicaciones científicas la incidencia de síntomas individuales dentro de un sindrome deberían ser precisados individualmente, indicando el número de los que lo padecen.

Los síndromes descritos son anormalidades funcionales para las cuales no se ha definido una causa concreta. Se presume que la valoración rutinaria (entrevista, examen físico y otras investigaciones apropiadas) ha excluido patología local obvia, tal como la de naturaleza infecciosa, neoplásica, hormonal o metabólica.

\section{Sindromes dolorosos genito-urinarios}

Son todos crónicos. El dolor es el sintoma principal, pero existen otras molestias concomitantes que provienen del tracto urinario inferior, intestino y genitales.

- El síndrome de dolor vesical es la manifestación de dolor suprapúbico relacionado con el llenado vesical, acompañado de otros síntomas como un incremento en la frecuencia diurna y nocturna, en ausencia de infección probada o cualquier otra patología ${ }^{9}$. (NUEVA)

- El síndrome de dolor uretral es el dolor uretral recurrente en la micción, con frecuencia diurna y nocturia, en ausencia de infección comprobada u otra patología. (NUEVA)

- El síndrome de dolor vulvar es el dolor vulvar persistente o recurrente episódico, que se relaciona bien con el ciclo miccional o bien con síntomas sugestivos de disfunción del tracto uri-

\footnotetext{
8 "Estranguria", "tenesmo vesical" y "disuria" tienen un significado incierto y no deben ser usados en la disfunción del tracto urinario inferior, excepto que se especifique un significado concreto. Disuria textualmente significa 'micción anómala', es usada correctamente en algunos países europeos. Sin embargo, se usa con frecuencia para describir la sensación de picazón/quemazón propia de la infección urinaria. Se aconseja utilizar en el futuro los términos descritos a continuación.

${ }^{9}$ La ICS cree que es preferible este término de sindrome de vejiga dolorosa a "cistitis intersticial". La cistitis intersticial es un diagnóstico específico y requiere confirmación mediante hallazgos cistoscópicos e histológicos típicos. En la investigación de la vejiga dolorosa puede ser necesario excluir el carcinoma vesical in situ, la endometriosis y otras.
} 
nario o disfunción sexual. No existe infección comprobada ni otra patologia ${ }^{10}$. (NUEVA)

- El síndrome de dolor vaginal es el dolor vaginal persistente o recurrente episódico que se asocia con síntomas sugestivos de disfunción del tracto urinario inferior o disfunción sexual. No existe infección urinaria comprobada ni otra patología.

- El síndrome de dolor escrotal es el dolor escrotal persistente o recurrente episódico que se asocia con síntomas sugestivos de disfunción sexual o del tracto urinario inferior. No existe orquiepididimitis probada ni otra patología.

- El síndrome de dolor perineal es el dolor pelviano persistente o recurrente asociado a síntomas sugestivos de disfunción del tracto urinario inferior, sexual, intestinal o ginecológico. No existe infección probada ni otra patología ${ }^{11}$. (NUEVA).

Sindromes de los sintomas sugestivos de disfunción del tracto urinario inferior

En la práctica clínica, después de la valoración individual de los síntomas y hallazgos físicos del tracto urinario inferior, de los resultados del análisis de orina y otras investigaciones indicadas, llegamos a un diagnóstico empírico que se usa habitualmente como base para el tratamiento inicial.

- Urgencia, con o sin incontinencia, usualmente asociada a frecuencia y nocturia, puede ser descrita como síndrome de la vejiga hiperactiva, síndrome de urgencia o síndrome de urgencia-frecuencia. (NUEVA)

Estas combinaciones de síntomas son sugestivas de hiperactividad del detrusor demostrable urodinámicamente, pero pueden ser debidas a otras formas de disfunción uretro-vesical. Estos términos pueden ser usados si no existe infección probada u otra patología.

- Sintomas del tracto urinario inferior sugestivos de obstrucción es un término usado cuando un varón se queja predominantemente de síntomas de vaciado en ausencia de infección u otra patología diferente de las posibles causas de obstrucción urinaria ${ }^{12}$. (NUEVA)

\section{SIGNOS SUGESTIVOS DE DISFUNCION DEL TRACTO URINARIO INFERIOR (DTUI)}

MEDIDA DE LA FRECUENCIA, SEVERIDAD E IMPACTO DE LOS SÍNTOMAS DEL TRACTO URINARIO INFERIOR

Al pedirle al paciente que registre las micciones y los síntomas ${ }^{13}$ durante un periodo de días nos proporciona una valiosa información. El registro de los eventos de la micción puede realizarse de tres formas diferentes:

- Horario miccional: registra la hora de las micciones, del día y la noche, durante un mínimo de 24 horas. (NUEVA)

- Hoja de Frecuencia/Volumen (HFV): registra el volumen micional así como la hora de cada micción, del día y la noche, durante un mínimo de 24 horas. (CAMBIADA)

- Diario miccional [bladder diary]: registra las horas de micción y los volúmenes miccionales. Se acompaña de otros datos como episodios de incontinencia, uso de absorbentes y otra información tal como la ingesta de líquidos, el grado de urgencia y el grado de incontinencia ${ }^{14}$. (NUEVA)

\footnotetext{
${ }^{10}$ La ICS sugiere abandonar el uso del término vulvodinia ya que conduce a confusión del síntoma simple de dolor con el sindrome.

${ }^{11}$ La ICS sugiere abandonar el uso en hombres del término prostatodinia (dolor prostático) ya que conduce a confusión entre un sintoma único y el sindrome.

12 Usualmente se cree que en las mujeres los síntomas de vaciado sugieren hipoactividad del detrusor antes que obstrucción del tracto de salida.

${ }^{13}$ Los cuestionarios validados son útiles para registrar lo síntomas, su frecuencia, severidad y molestias que producen, así como el impacto de los síntomas de tracto urinario inferior STUI sobre la calidad de vida (QoL) o (CdV). Debería especificarse el instrumento utilizado.

${ }^{14}$ Es útil pedirle al individuo que valore el volumen de líquido ingerido. Esto puede hacerse con precisión midiendo el volumen de cada bebida o por aproximación preguntando cuantas veces ha bebido en un periodo de 24 horas. Si el individuo toma una cantidad significativa de comida rica en agua (verduras, frutas, ensaladas) se producirá un incremento apreciable de diuresis. Si está tratándose, debe marcarse el momento de la toma de un diurético en la tabla o en el diario.
} 
Los siguientes datos pueden abstraerse de las hojas de frecuencia volumen y de los diarios miccionales:

- Frecuencia diurna es el número de micciones registradas durante las horas de vigilia desde la primera micción tras el despertar y levantarse en la mañana hasta la última micción antes de dormirse. (NUEVA)

- Nocturia es el número de micciones registradas durante un sueño nocturno: cada micción es precedida y seguida de un periodo de sueño. (NUEVA)

- Frecuencia de 24 horas es el número total de micciones diurnas y episodios de nocturia durante un periodo específico de 24 horas. (NUEVA)

- Diuresis de 24 horas se mide recogiendo toda la orina de 24 horas. (NUEVA)

Usualmente se comienza a contar después de la primera micción realizada tras levantarse por la mañana, y se completa cuando se anota la primera micción realizada tras levantarse a la mañana siguiente.

- Poliuria se define como la diuresis de más de 2,8 litros de orina en 24 horas en adultos. Puede ser útil fijarse en la diuresis durante tramos horarios más cortos [van Kerrebroeck, Abrams, Chaikin, Donovan, Fonda, Jackson, Jennum, Johnson, Lose, Mattiasson, Robertson, and Weiss2002] ${ }^{15}$ (NUEVA)

- Volumen nocturno de orina se define como el volumen total de orina evacuado entre el momento en que el individuo se acuesta con intención de dormir y el momento en que se despierta con la intención de levantarse. (NUEVA)

Se excluye la última micción de antes de acostarse pero se incluye la primera tras levantarse por la mañana.

- La poliuria nocturna está presente cuando la proporción nocturna (normalmente durante las 8 horas en las que el paciente permanece en la cama) de orina evacuada en 24 horas aumenta. (NUEVA)

La orina evacuada durante la noche excluye la última micción antes de dormir pero incluye la primera micción de la mañana ${ }^{16}$.

- Volumen miccional máximo es el mayor volumen de orina evacuado durante una única micción y se determina bien a partir de la tabla de frecuencia/volumen o bien a partir del diario miccional. (NUEVA)

Pueden precisarse los volúmenes miccionales máximo, mínimo y medio durante el periodo de registro $^{17}$.

\section{EL EXAMEN FÍsICO}

Es esencial en la valoración de todos los pacientes con disfunción del tracto urinario inferior. Debería incluir un examen abdominal, pelviano, perineal y a ser factible un examen neurológico. En los pacientes con disfunción del tracto urinario inferior de posible origen neurológico, es necesario realizar un examen neurológico más profundo.

\section{Abdominal}

La vejiga puede ser apreciada por palpación abdominal o por percusión suprapúbica. Presionar suprapúbicamente o durante el examen vaginal bimanual puede inducir deseo miccional.

\section{La inspección perineal/genital}

Permite la descripción de la piel, por ejemplo la presencia de atrofia o excoriación, cualquier característica anatómica anormal y la observación de la incontinencia.

- Incontinencia urinaria (el signo) se define como el escape de orina visto durante la exploración: puede ser uretral o extrauretral.

\footnotetext{
15 Las causas de poliuria son variadas y revisadas en otra parte pero incluye el exceso habitual de ingesta líquida. La cifra de 2,8 litros está basada en la micción de una persona de $70 \mathrm{Kg}$ a $40 \mathrm{ml} / \mathrm{kg} /$ día.

16 El rango normal de producción de orina difiere con la edad y los rangos normales aún no están definidos. Por lo tanto, la poliuria nocturna está presente cuando más del 20\% (adultos jóvenes) al 33\% (más de 65 años) es producida por la noche. Por lo tanto la definición precisa es dependiente de la edad.

17 El término "capacidad vesical funcional" ya no se recomienda ya que "volumen miccional” es más claro y menos confuso, en particular si se lo califica, p.ej.: "volumen miccional máximo". Si se usa el término capacidad vesical, ello implica que ha sido medida de alguna manera, aunque sea sólo mediante ecografía abdominal. En adultos los volúmenes miccionales varían considerablemente. En niños, el "volumen esperado" puede calcularse a partir de la fórmula (30+(edad en años x 30) en ml). Asumiendo que no existe orina residual este será igual a la "capacidad vesical esperada".
} 
- Incontinencia urinaria de esfuerzo es la observación de escape por la uretra, simultánea o sincrónica con el esfuerzo ${ }^{18}$. (CAMBIADA)

La pérdida con el esfuerzo se supone que es debida al incremento de la presión abdominal.

- Incontinencia extrauretral se define como la observación de escape de orina por otras vías diferentes a la uretra. (ORIGINAL)

- Incontinencia sin clasificar es la observación de escape de orina involuntario que no puede ser clasificado en una de las categorías ya descritas en base a los signos y sintomas. (NUEVA)

\section{El examen vaginal}

Permite la descripción de anormalidades anatómicas observables y palpables y la valoración de la función de la musculatura del suelo pelviano, como se describe en el informe de la ICS sobre el Prolapso de Órganos Pelvianos. Las definiciones dadas son versiones simplificadas de las definiciones de dicho informe [Bump, Mattiasson, Bo, Brubaker, DeLancey, Klarskov, Shull, and Smith 1996].

- El prolapso de órganos pelvianos se define como el descenso de uno o más de: la pared vaginal anterior, la pared vaginal posterior, y el ápex de la vagina (cérvix/útero) o cúpula tras la histerectomía. La ausencia de prolapso se define como estadio 0 del soporte pelviano; El prolapso puede ser estadiado desde un estadio I al estadio IV. (NUEVA)

El prolapso de órganos pelvianos puede asociarse a la incontinencia urinaria y a otras disfunciones del tracto urinario inferior. Ocasionalmente puede enmascarar la incontinencia urinaria.

- Prolapso de cara vaginal anterior se define como el descenso de la cara anterior de la vagina, la unión uretrovesical (un punto situado a $3 \mathrm{~cm}$ proximal al meato urinario externo) o cualquier punto anterior proximal a éste está a menos de $3 \mathrm{~cm}$ por encima del plano del himen. (CAMBIADA)
- Prolapso del segmento apical de la vagina se define como cualquier descenso de la cicatriz de la cúpula vaginal (tras la histerectomía) o cérvix, por debajo de un punto situado a $2 \mathrm{~cm}$ menos que la longitud total de la vagina por encima del plano de himen. (CAMBIADA)

- Prolapso de la pared vaginal posterior se define como cualquier descenso de la pared vaginal posterior, de forma que un punto medio de la pared posterior vaginal situado $3 \mathrm{~cm}$ por encima del nivel de himen o cualquier punto posterior proximal a ése, desciende a menos de $3 \mathrm{~cm}$ por encima del plano del himen. (CAMBIADA)

\section{Función muscular del suelo pelviano}

puede ser definida cualitativamente por el tono en reposo y por la fuerza de una contracción voluntaria o refleja en: fuerte, débil o ausente, o mediante un sistema de gradación validado (p.e.: el de Oxford 1-5). Una contracción del músculo pelviano puede ser valorada por inspección visual, por palpación, por electromiografía o por perineometría. Los factores a valorar incluyen fuerza, duración, desplazamiento y capacidad de repetición.

\section{Examen rectal}

permite la descripción de las anormalidades anatómicas observadas y palpables y es el método más sencillo de valorar la función de la musculatura pelviana en niños y hombres. El examen rectal es esencial en niños con incontinencia urinaria para excluir impactación fecal.

- función muscular del suelo pelviano puede ser definida cuantitativamente, durante el examen rectal, por el tono en reposo y por la fuerza de una contracción voluntaria, como fuerte, débil o ausente. (NUEVA)

\section{PRUEBA DEL ABSORBENTE}

Puede usarse para cuantificar la cantidad de orina perdida durante los episodios de incontinencia. Varía desde una prueba de provocación corta hasta la valoración de pérdidas en 24 horas.

\footnotetext{
${ }^{18}$ La tos puede inducir una contracción del detrusor, por lo tanto el signo de la incontinencia de esfuerzo sólo es un indicador fiable de incontinencia de esfuerzo urodinámica cuando el escape tiene lugar sincrónicamente a la tos (justo con el primer golpe de tos y que no continua tras el final de dicha tos).
} 


\section{HALLAGOS Y CONDICIONES URODINÁMICOS}

TÉCNICAS URODINÁMICAS

Existen dos métodos principales de investigaciones urodinámicas:

- Estudios urodinámicos convencionales que se realizan normalmente en el laboratorio de urodinámica y usualmente se hace con un llenado artificial. (NUEVA)

Llenado vesical artificial "no fisiológico", se define como el llenado de la vejiga, a través de un catéter, con un líquido y a un ritmo específico. (NUEVA)

- Estudios urodinámicos ambulatorios se definen como pruebas funcionales del tracto urinario inferior, utilizando un llenado fisiológico, mientras el sujeto realiza sus actividades habituales $^{19}$

Llenado vesical fisiológico significa que la vejiga se llena por diuresis y no por un medio artificial.

Tanto la cistomanometría de llenado como los estudios de presión/flujo de vaciado requieren las siguiente mediciones:

- Presión intravesical es la presión medida en el interior de la vejiga (ORIGINAL)

- Presión abdominal se refiere a la presión alrededor de la vejiga. En la práctica habitual se objetiva por la presión recogida a través del recto, vagina o menos frecuentemente por la presión extraperitoneal o de un estoma intestinal. La medición simultánea de la presión abdominal es esencial para la interpretación de la curva de presión intravesical. (ORIGINAL)

- Presión de detrusor es el componente de la presión intravesical generada por las fuerzas de la pared vesical (pasivas y activas). Se obtiene sustrayendo la presión abdominal de la intravesical. (ORIGINAL)

\section{CISTOMANOMETRÍA DE LLENADO}

La palabra "cistomanometría" (cistometry) se usa habitualmente para describir el estudio urodinámico de la fase de llenado del ciclo miccional. Para eliminar confusiones se proponen las siguientes definiciones:

- Cistomanometría de llenado es el método por el cual se mide la relación presión/volumen durante el llenado. (ORIGINAL)

La fase de llenado comienza al inicio del llenado y finaliza cuando el paciente y el "urodinamista" deciden la micción ${ }^{20}$.

Durante la fase de llenado es necesario definir la función uretral y del detrusor por separado.

La tasa a la que se llena la vejiga puede ser:

- Tasa de llenado fisiológica definida como una tasa de llenado menor que la tasa de llenado máxima previamente calculada - peso corporal máximo en $\mathrm{Kg}$ dividido por 4 expresado en $\mathrm{ml} / \mathrm{min}$ [Klevmark 1999] (CAMBIADA)

- Tasa de llenado no fisiológica se define como una tasa de llenado mayor que la tasa de llenado máxima previamente calculada - peso corporal máximo previamente calculado en $\mathrm{Kg}$ dividido por 4 expresado en $\mathrm{ml} / \mathrm{min}$ [Klevmark1999]. (CAMBIADA)

La función vesical de llenado debería describirse de acuerdo a la sensación vesical, actividad del detrusor, acomodación vesical y capacidad vesical ${ }^{21}$.

Sensación vesical durante la cistomanometría de llenado

- Sensación vesical normal puede ser evaluada en tres momentos definidos durante la cistomanometría de llenado y evaluados en relación con el volumen vesical en ese momento y en relación a las sensaciones percibidas por el paciente.

Primera sensación de llenado vesical es la sensación que tiene el paciente, durante la cistomanometría de llenado, cuando se hace consciente de que su vejiga se está llenando. (NUEVA)

\footnotetext{
${ }^{19}$ El término Urodinámica ambulatoria se usa para indicar que la monitorización tiene lugar fuera del laboratorio de urodinámica, y no para indicar la movilidad del sujeto usando un llenado natural.

${ }^{20}$ La ICS ya no considera oportuno dividir la tasa de llenado en lento, medio y rápido. En la práctica casi todos los estudios se realizan usando tasas de llenado medias y esto tiene un amplio rango. Puede ser más importante considerar durante las investigaciones si la tasa de llenado usada durante los estudios urodinámicos convencionales puede considerarse fisiológica.

${ }^{21}$ Mientras la sensación vesical se valora durante la cistomanometría de llenado, el asumir que la sensación proviene únicamente de la vejiga sin componentes uretrales o pelvianos puede ser falsa.
} 
Primer deseo miccional se define como la sensación, durante la cistomanometría de llenado, que decide al paciente a ir a orinar tan pronto como sea posible, pudiendo ser demorada si es necesario. (CAMBIADA)

Fuerte deseo miccional se define, durante la cistomanometría de llenado, como un deseo persistente sin miedo al escape de orina. (ORIGINAL)

- Sensación vesical aumentada se define, durante la cistomanometría de llenado, como un deseo temprano de orinar que ocurre a bajo volumen vesical y que persiste ${ }^{22}$. (NUEVA)

- Sensación vesical disminuida se define, durante la cistomanometría de llenado, como una sensación disminuida durante el llenado vesical. (NUEVA)

- Sensación vesical ausente significa que, durante la cistomanometría de llenado, el individuo no tiene sensación vesical. (NUEVA)

- Sensación vesical inespecífica, son aquellas otras que pueden hacer consciente al individuo de su llenado vesical, por ejemplo, plenitud abdominal o síntomas vegetativos. (NUEVA)

- Dolor vesical, es un término autoexplicado y es un hallazgo anormal. (NUEVA)

- Urgencia, es el súbito e irresistible deseo de orinar $^{23}$. (NUEVA)

- Umbral sensorial vesico/uretral, se define como la corriente de menor intensidad que produce sensación repetidamente y es simultánea a la estimulación eléctrica del lugar bajo investigación [Andersen, Blaivas, Cardozo, and Thüroff1992]. (ORIGINAL)
Función del detrusor durante la cistomanometría de llenado

El individuo es capaz de inhibir la actividad del detrusor hasta que puede realizar la micción. Cuando el paciente tiene deseo miccional, por lo tanto cuando el objetivo del estudio de llenado se ha conseguido, normalmente se le da "permiso para orinar" (ver cistomanometría de llenado pag. 13). Este momento se indica en el trazado urodinámico. Cualquier actividad del detrusor antes de este "permiso" se define como "actividad involuntaria del detrusor".

- Función normal del detrusor: el llenado vesical se produce con cambios de presión mínimos. No se producen contracciones fásicas del detrusor a pesar de las provocaciones. (ORIGINAL)

- Hiperactividad del detrusor es un hallazgo urodinámico caracterizado por contracciones involuntarias del detrusor durante la fase de llenado y que pueden ser espontáneas o provocadas $^{24}$. (CAMBIADA)

Existen ciertos patrones de hiperactividad del detrusor:

- Hiperactividad fásica del detrusor se define por una curva cistomanométrica característica que puede o no producir incontinencia urinaria $^{25}$. (NUEVA)

- Hiperactividad terminal del detrusor se define como una contracción involuntaria única que no puede ser suprimida, que ocurre a la capacidad cistomanométrica del estudio y que da como resultado una incontinencia que usualmente termina en una micción completa ${ }^{26}$. (NUEVA)

\footnotetext{
22 La valoración de la sensación vesical del sujeto es subjetiva y no es, por ejemplo, posible cuantificar "volumen vesical pequeño" en la definición de "sensación vesical aumentada".

${ }^{23}$ La ICS ya no recomienda el uso de los términos "urgencia motora” y "urgencia sensorial”. Estos términos a menudo son mal utilizados y tienen poco significado intuitivo. Además, puede ser simplista el relacionar justo el momento de la urgencia con la presencia o ausencia de hiperactividad del detrusor cuando usualmente hay una caída simultáneamente de la presión uretral. (version original integra: The ICS no longer recomends the terms "motor urgency" and "sensory urgency". These terms are often misused and have little intuitive meaning. Furthemore, it may be simplistic to relate urgency to the presence or absence of detrusor overactivity when there is usually a concomitant fall in urethral pressure.)

${ }^{24}$ No hay un limite inferior para la amplitud de una contracción involuntaria del detrusor pero la interpretación cierta de las existencia de ondas de contracción de baja presión (amplitud menor de $5 \mathrm{~cm}$ de $\mathrm{H}_{2} 0$ ) depende de una técnica urodinámica de "alta calidad". La frase "que el paciente no puede suprimir completamente" ha sido borrada de la antigua definición.

25 Las contracciones fásicas del detrusor no van siempre acompañadas de sensación. Pueden ser interpretadas como la primera sensación de llenado vesical, o como un deseo normal de micción.

26 "Hiperactividad terminal del detrusor" es un término nuevo de la ICS: se da asociada tipicamente a una sensación vesical disminuida, por ejemplo en el paciente anciano con accidente cerebro-vascular en el que la urgencia puede notarse en el momento en que ocurre la contracción micional. Sin embargo, en los paciente con lesión medular completa puede no haber sensación en absoluto.
} 
- Incontinencia por hiperactividad del detrusor es la incontinencia debida a una contracción involuntaria del detrusor. (NUEVA)

En un paciente con una sensación normal, la urgencia habitualmente se percibe justo antes del episodio de fuga ${ }^{27}$.

La hiperactividad neurogénica del detrusor puede ser calificada también de acuerdo con la causa cuando sea posible; por ejemplo:

- Hiperactividad del detrusor neurogénica cuando la causa de la disfunción es neurogénica.

Este término reemplaza al término "hiperreflexia del detrusor". (NUEVA)

- Hiperactividad idiopática del detrusor cuando no hay una causa definida. (NUEVA)

Este término reemplaza a "inestabilidad del detrrusor" 28

En la práctica clínica y de investigación, la amplitud de las evaluaciones neurológicas varía. Es probable que la proporción de hiperactividad del detrusor neurogénica/idiopática aumente si se realizan valoraciones neurológicas más completas.

Se observan otros patrones de hiperactividad del detrusor, por ejemplo, la combinación de hiperactividad del detrusor terminal y fásica, y las contracciones del detrusor mantenidas de alta presión que se ven en los pacientes con lesión medular cuando sus intentos miccionales coinciden con un esfinter disinérgico.

- Las maniobras de provocación (de estimulación) se definen como las técnicas usadas durante los estudios urodinámicos para desencadenar la hiperactividad del detrusor, por ejemplo, llenado rápido, uso de medio ácido o frío, cambios posturales y lavado de manos, etc. (NUEVA)
Acomodación vesical durante la cistomanometría de llenado

- La acomodación vesical describe la relación entre el cambio en el volumen vesical y el de la presión del detrusor ${ }^{29}$. (CAMBIADA)

La acomodación se calcula dividiendo el incremento de volumen $(\Delta \mathrm{V})$ entre el incremento en la presión de detrusor ( $\Delta$ Pdet) correspondiente a este incremento de volumen. Se expresa en $\mathrm{ml} / \mathrm{cm} \mathrm{H}_{2} \mathrm{O}$. (Acomodación $=\Delta \mathrm{V} / \Delta$ Pdet).

Se han descrito varios modos de calcular la acomodación vesical. La ICS recomienda que se usen dos puntos estándares para el cálculo de la acomodación: el investigador puede desear añadir puntos adicionales. Los puntos estándares son:

1. La presión de detrusor al inicio del llenado vesical y el correspondiente volumen inicial (usualmente cero), y

2. La presión de detrusor y el correspondiente volumen vesical a la capacidad cistomanométrica o inmediatamente antes del inicio de cualquier contracción del detrusor que cause fuga significativa (y por ello cause disminución del volumen vesical, afectando al cálculo de la acomodación). Ambos puntos se miden excluyendo cualquier contracción del detrusor.

Capacidad vesical: durante la cistomanometría de llenado

- Capacidad cistomanométrica, es el volumen vesical alcanzado al final del cistometrograma de llenado, cuando usualmente se da el "permiso de micción”. Se debería especificar el objetivo final, por ejemplo, si el llenado se detiene cuando el paciente alcanza un deseo miccional normal. La

\footnotetext{
${ }^{27}$ La ICS recomienda que los términos "incontinencia por urgencia motora" e "incontinencia refleja”, no se usen ya que no tienen un significado intuitivo_y son usados a menudo erróneamente (Versión integra en inglés: ICS recommends that the terms "motor urge incontinence" and "reflex incontinence", should no longer be used as they have no intuitive meaning and are often misused.)

${ }^{28}$ Los términos "inestabilidad del detrusor" e "hiperreflexia del detrusor" se usaron ambos como términos genéricos, en el lenguaje Inglés y en Escandinavia, antes del primer informe de la ICS de 1976. Como un compromiso se refirieron a hiperactividad idiopática y neurogénica respectivamente. Como no existe una lógica real o significado intuitivo para estos términos, la ICS cree que deberían ser abandonados.

${ }^{29}$ La observación de una acomodación disminuida durante la cistomanometría convencional se relaciona a menudo con el llenado relativamente rápido: la incidencia de acomodación disminuida es marcadamente más baja si la vejiga se llena a ritmo fisiológico, como en las urodinámicas ambulatorias.
} 
capacidad cistomanométrica es el volumen orinado más la orina residual ${ }^{30}$. (CAMBIADA)

- Capacidad cistomanométrica máxima, en pacientes con sensación normal, es el volumen al cual el paciente nota que ya no puede demorar más su micción (fuerte deseo miccional). (ORIGINAL)

- Capacidad vesical anestésica máxima es el volumen al que puede ser llenada una vejiga bajo anestesia general profunda o anestesia raquídea y debería ser calificada de acuerdo con el tipo de anestesia utilizada y la velocidad de infusión, tiempo de llenado y presión a la cual se ha llenado la vejiga. (CAMBIADA)

Función uretral durante la cistomanometría de llenado

El mecanismo de cierre uretral durante el llenado puede ser competente o incompetente.

- El mecanismo de cierre uretral normal mantiene una presión de cierre positiva durante el llenado vesical incluso en presencia de presión abdominal aumentada, aunque ésta pueda ser superada por hiperactividad del detrusor. (CAMBIADA)

- El mecanismo de cierre uretral incompetente se define como aquel que permite el escape de orina en ausencia de contracción del detrusor. (ORIGINAL)

- La incontinencia por relajación uretral se define como el escape debido a relajación uretral en ausencia de aumento de la presión abdominal o hiperactividad del detrusor ${ }^{31}$. (NUEVA)

- Incontinencia de esfuerzo urodinámica se observa durante el llenado cistomanométrico, y se define como el escape involuntario de orina du- rante el aumento de presión abdominal, en ausencia de contracción del detrusor. (CAMBIADA)

La incontinencia de esfuerzo urodinámica es el término actual preferido en vez de "Incontinencia genuina de esfuerzo".

Evaluación de la función uretral durante la cistomanometría de llenado ${ }^{32}$

- Medida de la presión uretral

- Presión uretral se define como la presión de fluido necesaria para abrir una uretra cerrada. (ORIGINAL)

- El perfil de presión uretral es la curva que indica la presión intraluminal a lo largo de la longitud uretral (ORIGINAL)

- Perfil de presión uretral de cierre viene dado por la sustracción a la presión uretral de la presión intravesical. (ORIGINAL)

- Presión uretral máxima es la máxima presión del perfil medido. (ORIGINAL)

- Presión de cierre uretral máxima (PCUM) es la máxima diferencia entre la presión uretral y la presión intravesical. (ORIGINAL)

- Longitud del perfil funcional es la longitud de la uretra a lo largo de la cual la presión uretral excede la presión intravesical en mujeres.

- Cociente de "transmisión" de presión es el incremento de la presión uretral bajo el esfuerzo expresado como un porcentaje del incremento de la presión intravesical medida simultáneamente.

- Presión abdominal de punto de fuga es la presión intravesical a la que se produce escape por un incremento de la presión abdominal en ausencia de contracción del detrusor ${ }^{33}$. (NUEVA)

\footnotetext{
30 En ciertos tipos de disfunción, la capacidad cistomanométrica no se puede definir en los mismos términos. En ausencia de sensación la capacidad cistomanométrica no se puede definir en los mismos términos y es el volumen al cual el clínico decide terminar el llenado. La razón (o razones) para terminar el llenado deberían definirse, p.ej.: alta presión de detrusor durante el llenado, gran volumen infundido o dolor. Si hay una micción no controlada, es el volumen al cual comienza ésta. En presencia de un esfinter incompetente la capacidad cistomanométrica puede ser aumentada significativamente mediante la oclusión de la uretra p.ej.: por un catéter de Foley.

31 Las fluctuaciones en la presión uretral se han definido como la "uretra inestable". Sin embargo, el significado de la fluctuaciones y el término en sí mismo carece de claridad y no se recomienda por la ICS. Si los síntomas se observan en asociación con un descenso en la presión uretral se debería ofrecer una descripción completa.

32 En las pacientes con incontinencia de esfuerzo, existe un espectro de características uretrales que va desde una uretra altamente móvil con una función intrínseca buena a una uretra inmóvil con función intrínseca pobre. Cualquier especificación en categorías tales como "hipermovilidad uretral" y "deficiencia esfinteriana intrínseca" puede ser simplista y arbitraria, y requiere más investigación.

${ }^{33} \mathrm{El}$ punto de presión de fuga o escape debería ser calificado de acuerdo con el lugar de medición de la presión (rectal, vaginal o intravesical) y el método por el cual se ha generado la presión (tos o Valsalva). Las presiones del punto de fuga pueden ser calculadas de tres formas a partir de diferentes valores de línea basal que son de uso habitual: - cero (el verdadero cero de la presión intravesical), - el valor de $\mathrm{p}_{\mathrm{ves}}$ medido al volumen vesical cero, o - el valor de la $\mathrm{p}_{\mathrm{ves}}$ inmediatamente antes de la tos o Valsalva (usualmente a 200 o $300 \mathrm{ml}$ de capacidad vesical). La línea basal usada y la presión de línea base, deberian especificarse.
} 
- Presión de punto de fuga del detrusor se define como la menor presión del detrusor que produce escape de orina en ausencia de contracción del mismo ni aumento de la presión abdominal $^{34}$. (NUEVA)

\section{ESTUDIOS DE PRESIÓN FLUJO}

La micción se describe según la función del detrusor y uretra y se valora mediante la medida del flujo urinario y las presiones de micción.

- Los estudios de presión/flujo son el método por el cual se mide la relación entre la presión vesical y el flujo urinario durante el vaciado vesical. (ORIGINAL)

La fase de vaciado (micción) comienza cuando se da el "permiso para orinar" o cuando se produce una micción incontrolada, y termina cuando el paciente considera que ha terminado de orinar.

\section{Flujometría}

El flujo urinario puede ser continuo o intermitente. Es intermitente cuando un individuo presenta inicios y paradas durante una micción. La curva de flujo continuo se define como una curva en forma de arco suave o fluctuante si hay múltiples picos ${ }^{35}$.

- Flujo se define como el volumen de orina expelido por la uretra por unidad de tiempo. Se expresa en $\mathrm{ml} / \mathrm{s}$ (ORIGINAL)

- Volumen miccional es el volumen total expelido vía uretral. (ORIGINAL)

- Flujo máximo es el máximo valor del flujo medido tras la corrección de artefactos. (CAM$B I A D A)$

- Tiempo de micción es la duración total de la micción, incluye las interrupciones. Cuando la micción se produce sin interrupción, el tiempo miccional es igual al tiempo de flujo. (ORIGINAL)

- El tiempo de flujo es el tiempo de la micción durante el cual se puede detectar flujo. (ORIGINAL)
- El flujo medio es el volumen miccional dividido por el tiempo de flujo. El flujo medio debería interpretarse con cuidado si el flujo es interrumpido o si hay un goteo terminal. (CAMBIADA)

- El tiempo al flujo máximo es el tiempo transcurrido desde el inicio del flujo al momento de flujo máximo. (ORIGINAL)

Medidas de presión durante los estudios de presión flujo (EPF)

Las medidas siguientes son aplicables a cada una de las curvas de presión: intravesical, abdominal y del detrusor.

- Presión premiccional es la presión registrada inmediatamente antes de la contracción inicial isovolumétrica. (ORIGINAL)

- Presión de apertura es la presión registrada al inicio del flujo miccional (considerar el tiempo de retardo o demora). (ORIGINAL)

- Tiempo de apertura es el tiempo de demora desde que se inicia la contracción del detrusor hasta el inicio del flujo. (ORIGINAL)

Este es el periodo de la micción de contracción isovolumétrica inicial. La demora en la medida del flujo debería tomarse en consideración cuando se mida el tiempo de apertura.

- Presión máxima es el máximo valor de presión medido. (ORIGINAL)

- Presión al flujo máximo es la presión más baja registrada en el momento de flujo máximo. (ORIGINAL)

- Presión de cierre es la presión medida al final del flujo. (ORIGINAL)

- La presión de micción mínima es la mínima presión registrada durante los periodos de flujo medible pero no es necesariamente igual a la presión de apertura o de cierre.

- La demora del flujo es el tiempo de demora entre un cambio en la presión de la vejiga y el correspondiente cambio en la medida del flujo.

Función del detrusor durante la micción

- Función del detrusor normal

La micción normal se consigue por contracción

\footnotetext{
${ }^{34}$ La presión de punto de fuga del detrusor se ha usado muy frecuentemente para predecir los problemas del tracto urinario superior en los pacientes neurológicos con acomodación vesical disminuida. La ICS la ha definido como "en ausencia de una contracción del detrusor" aunque otros la midieran durante las contracciones involuntarias del detrusor.

35 La forma exacta de la curva de flujo está condicionada por la contractilidad del detrusor, la presencia de cualquier esfuerzo abdominal y el tracto de salida uretral [Schafer, Abrams, Liao, Mattiasson, Pesce, Spangberg, Sterling, Zinner, and van Kerrebroeck2002].
} 
continua del detrusor, iniciada voluntariamente, que conduce al vaciado vesical completo en un periodo tiempo normal, en ausencia de obstrucción. Para una contracción del detrusor dada, la magnitud del aumento de presión registrada dependerá del grado de resistencia del tracto de salida. (ORIGINAL)

- Actividad anormal del detrusor puede ser subdividida en:

- Hipoactividad del detrusor se define como una contracción de fuerza y/o duración disminuida, que tiene como consecuencia un vaciado vesical prolongado y/o la imposibilidad de conseguir un vaciado vesical completo en un periodo de tiempo normal. (ORIGINAL)

- Detrusor acontráctil es aquel en el que no se logra mostrar ningún tipo de contracción durante los estudios urodinámicos ${ }^{36}$. (ORIGINAL). Sería correcto si se refiere al estudio realizado, pero no como diagnóstico, ya que la ausencia de contracción puede ser debida a inhibición psíquica (añadido en el seminario).

- El residuo postmiccional (RP) se define como el volumen de orina que queda en la vejiga al final de la micción ${ }^{37}$. (ORIGINAL)

\section{Función uretral durante la micción}

Durante la micción, la función uretral puede ser:

Normal: la uretra se abre, y se encuentra relajada continuamente para permitir a la vejiga vaciarse a una presión normal. (CAMBIADA)

Anormal: la obstrucción se debe, bien a hiperactividad uretral, o bien a que no puede abrirse debido a anormalidades anatómicas, tales como una próstata aumentada o una estenosis uretral.

- La obstrucción del tracto de salida vesical es una expresión genérica para denotar obstrucción durante el vaciado y se caracteriza por una presión de detrusor aumentada y un flujo urinario disminuido. Usualmente se diagnostica mediante el estudio de los valores sincrónicos del flujo (Q) y la presión del detrusor $\left(\mathrm{P}_{\text {det }}\right)^{39}$. (CAMBIADA)

- La micción disfuncional se caracteriza por un flujo intermitente y/o fluctuante debido a contracciones intermitentes involuntarias de la musculatura estriada peri-uretral durante la micción, en individuos neurológicamente normales ${ }^{39}$. (CAMBIADA)

La disinergia detrusor esfinter describe una contracción del detrusor simultánea a una contracción involuntaria de la musculatura estriada uretral y/o periuretral durante la contracción del detrusor. Ocasionalmente puede no aparecer flujo $^{40}$. (ORIGINAL)

- La obstrucción por no relajación del esfinter uretral ocurre usualmente en individuos con una lesión neurológica y se caracteriza por una uretra obstructiva que no se relaja y consecuentemente un flujo miccional disminuido ${ }^{41}$. (NUEVA)

\section{ALTERACIONES (Conditions)}

(Dada la dificultad de dar traducción a la palabra "conditions", que expresa el significado que tiene en inglés, es preferible mantenerla en este idioma hasta que encontremos mejor solución).

- La retención aguda de orina se define como

\footnotetext{
36 Ante una actividad contráctil normal del detrusor, según la resistencia del tracto de salida podemos encontrar: altas presiones del detrusor si la resistencia es elevada, presiones normales si la resistencia es normal o bajas presiones si la resistencia uretral es baja.

37 Si tras repetidas flujometrías libres no se demuestra orina residual, entonces el hallazgo de orina residual durante los estudios urodinámicos debería ser considerado como un artefacto, debido a las circunstancias de la prueba.

${ }^{38}$ La obstrucción del tracto de salida vesical ha sido definida para los varones pero hasta el momento, no lo ha sido adecuadamente para las mujeres y niños.

39 Aunque la micción disfuncional no es un término muy específico, se prefiere a términos como "vejiga neurogénica no-neurogénica". Pueden ser preferibles otros términos como: "disinergia detrusor-esfinter idiopática", o "disfunción miccional de hiperactividad esfinteriana”. Sin embargo, el término micción disfuncional está bien establecido. La alteración se da más frecuentemente en niños. Aunque se cree que la etiología son las contracciones del suelo pelviano, es posible que también juegue un papel importante la musculatura estriada intra-uretral.

${ }^{40}$ La disinergia detrusor esfinter ocurre típicamente en pacientes con una lesión suprasacra, tras una lesión medular alta y es rara en lesiones medulares bajas. Aunque la musculatura estriada periuretral e intrauretral se cree usualmente responsable, la musculatura lisa del cuello vesical o la uretra puede también serlo.

${ }^{41}$ La obstrucción por no relajación esfinteriana se encuentra en lesiones medulares sacras e infrasacras como en el mielomeningocele, y tras cirugía radical pelviana. Además a menudo hay incontinencia de esfuerzo urodinámica durante el llenado vesical. Este término reemplaza a "obstrucción aislada del esfinter distal".
} 
una vejiga dolorosa, palpable o percutible, cuando el paciente es incapaz de orinar ${ }^{42}$. (NUEVA)

- La retención crónica de orina se define como una vejiga no dolorosa, que permanece palpable o percutible incluso una vez que el paciente ha orinado. Estos pacientes pueden ser incontinentes $^{43}$. (NUEVA)

- La obstrucción benigna prostática es una forma de obstrucción del TUI, y puede diagnosticarse cuando la causa de la obstrucción es un agrandamiento prostático benigno por la hiperplasia benigna prostática. (NUEVA)

- La hiperplasia prostática benigna es un término usado (y reservado para) el patrón histológico típico que define la enfermedad. (NUEVA)

- El agrandamiento prostático benigno se define como el aumento prostático debido a la histología de hiperplasia prostática benigna. El término "agrandamiento prostático" debería usarse en ausencia de histología prostática. (NUEVA)

\section{TRATAMIENTO}

Las siguientes definiciones fueron publicadas en el $7^{\circ}$ informe de la ICS sobre técnicas de rehabilitación del tracto urinario inferior [Andersen, Blaivas, Cardozo, and Thüroff1992] y aún mantiene su forma original.

\section{LA REHABILITACIÓN DEL TRACTO URINARIO INFERIOR}

se define como un tratamiento no-quirúrgico y no-farmacológico para normalizar la función del tracto urinario inferior e incluye:

- Entrenamiento del suelo pelviano: definido como contracción y relajación voluntaria, selectiva y repetitiva de músculos específicos del suelo pelviano.
- La retroalimentación (o biofeedback): es la técnica por la cual la información sobre un proceso fisiológico normalmente inconsciente se hace consciente al paciente y/o al terapeuta como una señal visual, auditiva o táctil.

- La modificación de conducta se define como el análisis y cambio de las relaciones entre los sintomas del paciente y su entorno para el tratamiento de patrones miccionales mal-ajustados.

Esto puede ser conseguido por modificación de la conducta y/o el entorno del paciente.

\section{LA ESTIMULACIÓN ELÉCTRICA}

Es la aplicación de la corriente eléctrica al estímulo de las vísceras pelvianas o sus nervios aferentes.

El objetivo de la estimulación eléctrica puede ser inducir una respuesta terapéutica directamente o modular el tracto urinario inferior, intestino o disfunción sexual.

\section{EL CATETERISMO}

Es una técnica para el vaciado vesical empleando un catéter para drenar la vejiga o un reservorio urinario.

\section{Cateterismo intermitente}

Se define como el drenaje de la vejiga o reservorio urinario con la retirada posterior del catéter.

Se definen los siguientes tipos de cateterismo intermitente:

- Autocateterismo intermitente: realizado por el propio paciente.

- Cateterismo intermitente por un cuidador (p.ej.: médico, enfermera o familiar).

- Cateterismo intermitente limpio: usa una técnica limpia. Esto implica el uso de técnicas habituales de lavado y el uso de catéteres de un solo uso o reutilizables lavados.

\footnotetext{
42 Aunque habitualmente se piensa en la retención aguda como dolorosa, en ciertas circunstancias el dolor puede no ser una característica de presentación, por ejemplo cuando es debida a un disco intervertebral prolapsado, por parto, tras anestesia regional con una anestesia epidural. El volumen de retención debería ser significativamente mayor que el esperado para la capacidad vesical normal. En pacientes post quirúrgicos, debido a vendajes del abdomen inferior, puede ser dificil detectar una vejiga dolorosa, palpable o percutible.

43 La ICS ya no recomienda el uso del término "incontinencia por rebosamiento". Este término se considera confuso y carente de una definición convincente. Si se usa, debería exponerse una definición precisa y una fisiopatología asociada, como una función uretral disminuida, o una hiperactividad vesical/acomodación vesical disminuida. El término de retención crónica, excluye una dificultad de vaciado transitoria, por ejemplo tras una cirugía por incontinencia de esfuerzo, e implica una orina residual significativa; se ha mencionado previamente una cifra mínima de $300 \mathrm{ml}$.
} 
- Cateterismo intermitente aséptico: usa una técnica estéril. Ello implica una desinfección genital, el uso de catéteres, guantes y otros instrumentos estériles.

\section{Cateterismo permanente}

Es cuando se deja un catéter que permanece en la vejiga, reservorio urinario o conducto ileal por un periodo mayor al intervalo entre dos micciones.

\section{DISPARO DEL REFLEJO VESICAL}

Comprende las maniobras realizadas por el paciente o el terapeuta con el objeto de provocar una contracción refleja del detrusor mediante un estímulo exteroceptivo.

Las maniobras más comúnmente usadas son la percusión suprapúbica, frotación en cara interna del muslo y manipulación anorectal.

\section{COMPRESIÓN VESICAL}

Comprende diferentes maniobras con el ánimo de aumentar la presión intravesical y el objetivo de facilitar el vaciado vesical.

Las maniobras más comúnmente usadas son los esfuerzos abdominales: maniobra de Valsalva y maniobra de Credé.

\section{BIBLIOGRAFÍA (Formato del trabajo original)}

Abrams P, Blaivas JG, Stanton SL, Andersen JT. The standardisation of terminology of lower urinary tract function. The International Continence Society Committee on Standardisation of Terminology. Scand J Urol Nephrol Suppl 1988; 114:5-19.

Abrams P, Blaivas JG, Stanton SL, Andersen JT. Sixth report on the standardisation of terminology of lower urinary tract function. Procedures related to neurophysiological investigations: electromyography, nerve conduction studies, reflex latencies, evoked potentials and sensory testing. The International Continence Society Committee on Standardisation of Terminology, New York, May 1985. Scand J Urol Nephrol 1986; 20:161-164.

Andersen JT, Blaivas JG, Cardozo L, Thüroff J. ICS 7th report on the standardisation of terminology of lower urinary tract function: lower urinary tract rehabilitation techniques. Neurourol Urodyn 1992;11: 593-603.

Bump RC, Mattiasson A, Bo K, Brubaker LP, De Lancey JL, Klarskav P, Shull BL, Smith AB. The standardization of terminology of female pelvic organ prolapse and pelvic floor dysfunction. Am J Obstet Gynecol 1996;175:10-17.
Fonda D, Resnick NM, Colling J, Burgio K, Ouslander J, Norton C, Ekelund P, Versi E, Mattlasson A. Outcome measures for research of lower urinary tract dysfunction in frail and older people. Neurourol Urodyn 1998;17:273-281.

Griffiths D, Höfner K, van Mastrigt R, Rollema HJ, Spangberg A, Gleason D. Standardization of terminology of lower urinary tract function: pressure-flow studies of voiding, urethral resistance, and urethral obstruction. International Continence Society Subcommittee on Standardization of Terminology of Pressure-Flow Studies. Neurourol Urodyn 1997;16:1-18.

Klevmark B. Natural pressure-volume curves and conventional cystometry. Scand J Urol Nephrol Suppl 1999;201:1-4.

Lose G, Fantl JA, Victor A, Walter S, Wellas TL, Wyman J, Mattiasson A. Outcome measures for research in adult women with symptoms of lower urinary tract dysfunction. Neurourol Urodyn 1998; 17:255-262.

Lose G, Griffiths D, Hosker G, Kulseg-Hanssen S, Peruchini S, Schäfer W, Thind P, Versi E. Standardisation of urethral pressure measurement: report from the Standardisation Sub-Committee of the International Continence Society Neurourol Urodyn 2002;21:258-260.

Mattiasson A, Djurhuus JC, Fonda D, Lose G, Nordling J, Stohrer M. Standardization of outcome studies in patients with lower urinary tract dysfunction: a report on general principles from the Standardisation Committee of the International Continence Society Neurourol Urodyn 1998;17:249-253.

Nordling J, Abrams P, Ameda K, Andersen JT, Donovan J, Griffiths D, Kobayashi S, Koyanagui T, Shäfor W, Yalla S, Mattiason A. Outcome measures for research in treatment of adult males with symptoms of lower urinary tract dysfunction. Neurourol Urodyn 1998; 17:263-271.

OMS. Clasificación internacional del funcionamiento, de la discapacidad y de la salud (CIF-2). Website http://www.who. int/icidh. 24-10-2001.

Rowan D (chair), James ED, Kramer AE, Sterling AM, Suhel PF. Urodynamic equipment: technical aspects. Produced by the International Continence Society Working Party on Urodynamic Equipment. J Med Eng Technol 1987;11:57-64.

Schafer W, Abrams P, Liao L, Spanberg A, Pesce F, Zinner R, van Kerrebroeck P, Abrams P, Mattiasson A. Good urodynamic practices: uroflowmetry, filling cystometry, and pressure-flow studies. Neurourol Urodyn 2002;21:261-274.

Stohrer M, Goepel M, Kondo A, Kramer G, Madersbacher H, Millar R, Rosier A, Wyndaele JJ. The standardization of terminology in neurogenic lower urinary tract dysfunction: with suggestions for diagnostic procedures. International Continence Society Standardization Committee. Neurourol.Urodyn. 1999; 18:139-158.

Van Kerrebroeck P, Abrams P, Chaikin D, Donovan J, Fonda D, Jackson S, Jennum P, Johnson T, Lose G, Mattiasson A, Robertson G, Weiss J. The standardisation of terminology in nocturia: report from the Standardisation Sub-committee of the International Continence Society Neurourol Urodyn 2002;21:179-183.

Van Waalwijk, van Doorn E, Anders K, Khullar V, Kulseng-Hansen S, Pesce F, Robertson A, Rosario D, Schäfer W. Standardisation of ambulatory urodynamic monitoring: Report of the Standardisation Sub-Committee of the International Continence Society for Ambulatory Urodynamic Studies Neurourol Urodyn. 2000;19:113-125. 